\title{
EUTANASIA Y JUSTICIA DISTRIBUTIVA SANITARIA: APORTES A LA DISCUSIÓN MORAL SOBRE EL DERECHO A LA BUENA MUERTE
}

\author{
EUTHANASIA AND HEALTH DISTRIBUTIVE JUSTICE: \\ CONTRIBUTIONS TO MORAL DISCUSSION ON THE RIGHT \\ TO GOOD DEATH
}

ALEJANDRA ZÚÑIGA FAJURI ${ }^{1 *}$

\section{Resumen}

En este artículo se analizan algunos de los argumentos morales relacionados con la justa distribución de recursos sanitarios escasos que permiten discutir los problemas asociados a las demandas por la disponibilidad de la propia vida y al proyecto de ley que pretende despenalizar la eutanasia y el auxilio al suicidio en Chile.

\section{Palabras clave}

Eutanasia, justicia distributiva, recursos escasos.

\section{Abstract}

This paper reviews moral arguments related to the distribution of scarce health resources and the bioethical problems linked to a new law on Physician-Assisted Suicide and Euthanasia in Chile.

$1 \quad{ }_{1}^{*}$ Abogada y Doctora en Derecho. Profesora de la Escuela de Derecho de la Universidad de Valparaíso. Investigadora del Centro de Investigaciones de Filosofía del Derecho y Derecho Penal (CIFDE), Universidad de Valparaíso, Errázuriz 2120, Valparaíso, Chile. alejandra.zuniga@uv.cl. 


\section{Key words}

Euthanasia, distributive justice, limited resources.

\section{Introducción}

Durante los último 30 años se ha desarrollado una abundante literatura jurídica, bioética y filosófica destinada a defender la despenalización de la eutanasia activa y el auxilio médico al suicidio tanto en Chile como en el resto del mundo. Los argumentos esgrimidos van desde la defensa de los valores democráticos de la tolerancia, el pluralismo y el reconocimiento del derecho de las personas a una "muerte digna", hasta aquellos que se concentran en los dilemas bioéticos asociados a una tecnología capaz de mantener con vida, por tiempo indefinido, a personas que son víctimas de enfermedades incurables que provocan grandes sufrimientos. ${ }^{2}$

Este trabajo propone aportar a la discusión revisando argumentos bioéticos adicionales a los concernientes a la defensa de la autonomía moral. En particular, se analizan los dilemas de justicia distributiva detrás de la adjudicación de recursos sanitarios escasos. En esta línea, me ocupo de dos materias relacionadas con la distribución. Primero: ¿Responde la despenalización de la eutanasia a demandas de justicia distributiva sanitaria? Dicho de otro modo ¿transformar la eutanasia en un derecho básico, incorporándolo al mínimo sanitario como Garantía Explícita en Salud, logra una adjudicación justa de recursos sanitarios escasos? Segundo: ¿Requiere la despenalización de la eutanasia, de la distribución previa de otros recursos sanitarios escasos, como el acceso equitativo a cuidado paliativo, por ejemplo? Para responder a ambas preguntas vamos a repasar algunos de los principios de justicia distributiva del liberalismo igualitario y del utilitarismo, en particular, aquellos desarrollados por la economía de la salud y por la Teoría de la Justicia sanitaria de Norman Daniels.

\section{LA “BUENA MUERTE” Y EL MÍNIMO SANITARIO}

1. Primero, aclaremos los términos del debate. Tom Beauchamp, destacado bioeticista, define el Auxilio Médico al Suicidio (AMS) como la

2 Sobre los argumentos morales, normativa y jurisprudencia sobre eutanasia, véase ZÚÑIGA FAJURI, A.: "Derechos del paciente y eutanasia en Chile". En: Revista de derecho, UACH, Vol. XXI N² 2, 2008. pp. 111-130; ZÚÑIGA FAJURI, A.: "La justicia frente a las decisiones médicas”. En: Revista de Ciencias Sociales, N 55, 2009. pp. 203-232. 
elección voluntaria de la muerte por parte de un paciente con la asistencia de un médico. ${ }^{3}$ La Asociación Médica Mundial identifica al AMS como "proporcionar intencionalmente a una persona el conocimiento, los medios o ambos, para que cometa suicidio, incluyendo asesoramiento sobre dosis letales de medicamentos y la prescripción de dichas dosis letales". ${ }^{4}$ En otras palabras, el médico proporciona los medios para la muerte, pero el paciente administra el medicamento letal. El término eutanasia también se ha definido como la realización, a sabiendas e intencional, de un acto o práctica que pone fin a la vida de una persona a objeto de liberarla de una enfermedad incurable, un sufrimiento intolerable o una muerte indigna. La eutanasia se distingue del AMS por el hecho de que el médico causa directamente la muerte del paciente mediante la administración de una inyección letal o mediante la suspensión de tratamientos para provocar su muerte del paciente. ${ }^{5}$

Finalmente, la eutanasia activa implica un acto en relación con el fin de la vida, en cambio será pasiva o por omisión cuando se deje de hacer algo que es vital para la supervivencia del paciente. Por lo tanto, al omitir el tratamiento, el soporte vital o la reanimación, el médico teóricamente permite que el paciente muera naturalmente. En estos casos se hace una distinción importante entre la supervisión o rechazo de tratamiento y la asistencia médica al suicidio. ${ }^{6}$

2. ¿Transformar la eutanasia en un derecho básico, incorporándolo al mínimo sanitario como Garantía Explícita en Salud, logra una adjudicación justa de recursos sanitarios escasos? Tenemos que recurrir a los criterios de distribución de recursos sanitarios de al menos dos teorías de la justicia para responder a la cuestión de si es justo transformar a la eutanasia y el auxilio médico al suicidio en una Garantía Explícita en Salud. ${ }^{7}$

3 BEAUCHAMP, WALTERS, KHAN y MASTROIANNI: Contemporary Issues in Bioethics (7th ed.). Thomas Wadsworth, New York, 2008.

4 WORLD MEDICAL ASSOCIATION: Medical Ethics Manual, 2009. Disponible en: http://www.wma.net/en/30publications/30ethicsmanual/pdf/ethics_manu al_en.pdf.

5 KEOWN, J.: Euthanasia Examined (1st ed.). Cambridge University Press, Cambridge, 2004.

6 BEEAUCHAMP. TL y CHILDRESS. J.: Principles of Biomedical Ethics (6th ed.). Oxford University Press, New York, 2009.

$7 \quad$ El llamado "mínimo sanitario" en Chile está formado por aquellas prestaciones sanitarias -las enfermedades AUGE/GES- dotadas de las garantías de accesos, oportunidad, calidad y cobertura financiera. 
Para la Teoría de la Justicia del Liberalismo Igualitario -desarrollada fundamentalmente en la obra de John Rawls y aplicada al ámbito de la salud por autores como Norman Daniels, Ronald Dworkin, Elizabeth Anderson, ${ }^{8}$ entre otros- los bienes deben distribuirse conforme a ciertas razones relevantes que tienen relación con el significado esencial de los bienes y con su capacidad para promover la justa igualdad de oportunidades. ${ }^{9}$ Esto da por hecho que el Estado tiene un deber de prestación de índole redistributiva a objeto de garantizar que los menos aventajados -aquellas personas que han "perdido" en la lotería natural y/o social- puedan sin embargo participar en la vida social y alcanzar el mayor bienestar posible. ${ }^{10}$

¿Ahora, de qué modo decidiremos cuál es un nivel justo y suficiente de atención sanitaria accesible para todos, incluidos los más pobres? Teniendo presente la necesidad de racionalización del gasto en salud, Dworkin analiza los problemas asociados al llamado principio del rescate que defiende que la vida es el bien más importante y que todo lo demás debe ser sacrificado en su nombre de modo que, frente a la pregunta crucial sobre cuánto gastar, responderá: "hay que gastar todo lo que se tenga". El problema de este principio es que no se cuestiona cómo su uso puede dañar fatalmente la provisión de otros bienes sociales también importantes. Además, que no es racional gastar todo en salud si no se gana, a cambio, prácticamente nada en expectativa de vida. "Evidentemente ninguna sociedad sana puede hacer eso y ninguna persona individual organizaría su vida en torno a ese principio". ${ }^{11}$

Como alternativa al principio del rescate Dworkin propone el "Principio del seguro prudente" que, para determinar el mínimo sanitario garantizable razona teniendo presente el costo de oportunidad de los recursos sanitarios. Luego, las reglas que seguramente se seleccionarían incluyen la racionalización de los recursos y su uso respetando los planes de vida autónomos de las personas. Si bien no podemos saber que elegirá cada cual, sí podemos -a efectos de construir fundadamente un mínimo sanitario para toda la sociedad- formarnos algún juicio sobre las necesidades y preferencias generales de las personas. Por ejemplo, para la mayoría resultará irracional -por los costos de oportunidad -costear un seguro muy

8 ANDERSON, Elizabeth S.: "What Is the Point of Equality?". En: Ethics 109, 1999.

9 DANIELS, Norman: Just Health Care. Cambridge University Press, New York.1985.

10 DWORKIN, Ronald: Sovereign Virtue. The Theory and Practice of Equality. Harvard University Press, London. 2000.

11 Íbid, DWORKIN, 2000, p. 309. 
caro que cubra los gastos de un estado vegetativo persistente. Se considera preferible invertir en mejorar la vida consciente, garantizando un cuidado en condiciones dignas que no limite sus planes de vida. ${ }^{12}$

La sociedad organizada habrá, entonces, de garantizar un mínimo sanitario prudente que cubra un tratamiento médico ordinario - como el que garantiza en Chile el AUGE- y que se distribuya, dentro de ese mínimo, en base a la "necesidad" médica. Así, al entregar cuidado sanitario y permitir el funcionamiento normal de las personas, se asegura lo indispensable para lograr el propio plan de vida y participar en igualdad de condiciones en la vida pública. Se puede, entonces, defender como esencialmente injusto la definición de un mínimo sanitario en base a otros requisitos, como, por ejemplo, la capacidad de pago del enfermo. ${ }^{13}$

En fin, el Liberalismo Igualitario responderá a nuestra primera pregunta afirmativamente, defendiendo que sí es justo garantizar a todas las personas igual acceso a servicios sanitarios para lograr una "buena muerte", de modo que será mejor -será más equitativo- aquel sistema sanitario que asegura que la buena muerte no sea un privilegio sólo para quienes tienen recursos para financiar un viaje al extranjero o atención médica en una clínica privada que esté dispuesta a burlar la ley a cambio de una recompensa económica.

3. Avancemos ahora hacia la respuesta que eventualmente daría -a nuestra pregunta- la Teoría de la Justicia del Utilitarismo. Como se sabe, el Utilitarismo evalúa la moralidad de un acto en atención a su capacidad para producir el mejor resultado posible, el mayor bienestar, para el mayor número de personas. ${ }^{14}$ Lo más seductor de la teoría bienestarista es que la búsqueda de la utilidad debe llevarse a cabo de manera imparcial. "Seamos o no hijos de dios, tengamos o no un alma o una voluntad libre, la cuestión radical para esta teoría es que todos somos capaces de sufrimiento o felicidad, todos podemos estar mejor o peor en la vida por lo que, con

12 Íbid, DWORKIN, 2000.

13 Para B. WILLIAMS, es "un estado de cosas irracional" aquel en que algunas personas, teniendo las mismas necesidades que otras, no reciben el mismo tratamiento, siendo que el criterio de base para la adjudicación de recursos es el de la necesidad sanitaria. WILLIAMS, Bernard: “The Idea of Equality”. En: Problems of the Self. Cambridge University Press, 1973.

14 BENTHAM, Jeremy: An Introduction to the Principles of Moral and Legislation. Eds. Burns y Hart, Methuen, London. 1982 
independencia de cuales sean nuestras creencias, no podemos negar que la felicidad es algo valioso en la vida de cualquiera". ${ }^{15}$

A su vez, el utilitarismo es consecuencialista es la medida en que no admite realizar prohibiciones morales en base a razones arbitrarias sino que, en cambio, obliga a todo el que condene algo como moralmente incorrecto a que muestre a quién se perjudica con ese acto, es decir, se tiene que demostrar de qué modo la vida de alguien resulta empeorada a consecuencia de cierta conducta. De este modo, su método para resolver las cuestiones morales más difíciles será medir, de manera imparcial y racional, los cambios en el bienestar de las personas. Estas dos reglas -que se adecúan a nuestras intuiciones acerca de la importancia del bienestar de la humanidad y acerca de la necesidad de que las reglas morales se analicen a partir de sus consecuencias sobre dicho bienestar- permiten concluir que, si el bienestar de las personas es el bien del cual se ocupa la moral, entonces, el mejor acto moral será aquel que logre el máximo desarrollo del bienestar de la humanidad otorgando igual consideración al bienestar de cada cual.

La vertiente utilitarista del análisis sobre adjudicación de recursos sanitarios, llamada "Economía de la Salud", es una herramienta indispensable y extraordinariamente vigente cuando se discute el problema bioético de la distribución y racionamiento de recursos sanitarios escasos. El desafío de los actuales sistemas sanitarios -y el chileno no es una excepción- es enfrentar una creciente demanda de recursos en razón del envejecimiento de la población y de los extraordinarios avances de la medicina. Esto nos obliga a considerar seriamente el costo de oportunidad que significa proveer un cuidado sanitario de alto costo y bajo beneficio cuando existen tantas ganancias alternativas que pueden entregarse con esos mismos recursos, no sólo asignándolo para cubrir otras necesidades sanitarias, sino que para enfrentar otras responsabilidades públicas importantes como la educación, pensiones, trabajo, vivienda, etc.

Para que el cuidado sanitario no genere un costo social intolerable, el Utilitarismo propone técnicas de racionamiento capaces de evaluar la "eficiencia" de cada prestación sanitaria por medio de los análisis de costoefectividad (Cost Effectiveness Analysis) que es un tipo de evaluación económica que permite comparar distintas intervenciones de salud, en la que los efectos sobre los recursos se expresan en unidades monetarias y los efectos sobre la salud se expresan en unidades específicas no monetarias

15 KYMLICKA, Will: Contemporary Political Philosophy, an Introduction. Second Edition. Oxford University Press, 2002. P.11. 
de efectividad, por ejemplo, el número de vidas salvadas o el número de días libres de enfermedad. En los resultados de un análisis de costo efectividad, los costos netos están relacionados con un solo indicador de efectividad por lo que se requiere que los principales resultados de todas las opciones relevantes puedan ser expresados mediante el mismo indicador de efectividad, aunque dicho indicador pueda tomar para cada opción una magnitud diferente (como los AVAC). ${ }^{16}$

En resumen, si acordamos que es un principio abstracto de racionalidad práctica intentar alcanzar más de aquello que se considera valioso -pues las mejoras en la eficiencia productiva posibilitan incrementos de bienestary que para ser eficientes es imprescindible tener una cierta capacidad de racionalizar recursos, habremos de concluir que resulta inevitable poner límites a lo que podría hacerse para salvar vidas. Los recursos limitados de los Estados les obligan a hacer elecciones que van más allá de la mantención de la vida o la salud por lo que, para responder a la pregunta de si es justo y eficiente incorporar la eutanasia al mínimo sanitario, tenemos que considerar el siguiente racionamiento: en principio no parece justo gastar enormes cantidades de recursos a fin de mantener a toda costa la vida de una persona contra su voluntad. Estos recursos debieran, en cambio, adjudicarse eficientemente para asegurar tratamientos queridos por el paciente.

La mantención artificial y no querida de una vida de escasa calidad es un injusto, desde el punto de vista del utilitarismo. Conceder, en cambio, una "buena muerte" a quien lo solicita, es lo moralmente correcto y nos permite afirmar la necesidad de despenalizar la eutanasia y el auxilio médico al suicidio.

\section{Los cuidados paliativos y la eutanasia}

Finalmente, tratemos de responder a la segunda pregunta que nos hicimos al comienzo, cual es ¿Requiere la despenalización de la eutanasia de la distribución previa de otros recursos sanitarios escasos, como el acceso equitativo a cuidado paliativo, por ejemplo? Parece claro, a estas alturas, que para adjudicar correctamente recursos escasos debemos, al menos, considerar tanto el principio de necesidad como el principio de

16 GALVEZ GONZALEZ, Ana María (2004): "Guía metodológica para la evaluación económica en salud: Cuba 2003". En: Revista Cubana de Salud Pública. Enero-marzo. Vol. $30, \mathrm{~N}^{\circ} 1$. 
eficiencia. Es decir, debemos tener cuidado de utilizar únicamente criterios de eficiencia para la racionalización de los recursos pues los argumentos clásicos de los economistas -nos recuerda Charles Fried- parecen más los de quienes "saben el precio de todo y el valor de nada". ${ }^{17}$

$\mathrm{Si}$ consideramos que lo que queremos despenalizar es, evidentemente, la eutanasia voluntaria, resulta imprescindible que el Estado se comprometa a garantizar las condiciones mínimas necesarias para asegurar que la elección del paciente sea realmente voluntaria y, para ello, el sistema sanitario debe erradicar, en la medida de lo posible, todas aquellas situaciones de desigualdad sanitaria que puedan comprometer la autonomía del paciente. De este modo, debiera estar garantizado, a lo menos, un cuidado paliativo óptimo y atención de salud mental, cuando se requiera.

Recordemos que las principales críticas a la despenalización de la eutanasia se fundan en su eventual efecto tanto en el personal médico como en el propio paciente y su familia. En el primer caso, se teme que los más pobres y enfermos sean descuidados al verse "socavado" el compromiso de médicos y enfermeros con la vida. Se arguye, también, que la eutanasia pueda convertirse en una forma "rentable" de tratar a los enfermos terminales, que desaliente la búsqueda de nuevas curas y tratamientos para ellos y que desmotive el buen cuidado y alivio del dolor a los moribundos. Por otra parte, se sostiene que la eutanasia expone a las personas vulnerables a una fuerte presión para terminar con sus vidas a fin de liberar recursos médicos y dejar de ser una "carga" para sus familias. Incluso, los pacientes que son abandonados por sus familias pueden sentir que la eutanasia es la única solución.

Para responder a estas legítimas aprensiones es imprescindible que se garantice que el paciente reciba la mejor atención médica posible a fin de aliviar su sufrimiento. El Sistema Sanitario debe poner a disposición del paciente el cuidado paliativo necesario para que pueda descartarse que la solicitud de eutanasia sea, en realidad, un "grito de ayuda" ante síntomas o temores que pueden manejarse. Es preciso evitar que el paciente tome decisiones fundadas en una depresión evitable -no directamente relacionada con la depresión natural debido a la enfermedad-, o que opte por la eutanasia confundido y en un estado de incapacidad para hacer juicios sensatos.

$17 \quad$ FRIED, Charles: "Rights and Health Care. Beyond Equity and Efficiency". En: New England Journal of Medicine, Vol. 293, N 5.1975. 
El cumplimiento cabal de las normas de la Ley de Derechos del Paciente sobre cuidado paliativo - 20584 artículo 16- por todo el sistema sanitario público y privado y el perfeccionamiento y mejoramiento de las condiciones en las que viven los enfermos terminales o aquellos víctimas de grandes sufrimientos, permitirá descartar el uso indebido de la eutanasia por parte de paciente que se sienten vulnerables, una carga inútil y que temen que su enfermedad genere una angustia insoportable para su familia por falta o escasez de recursos. Hay que garantizar que la decisión sobre la eutanasia sea el resultado de un proceso bien diseñado que entregue un cuidado paliativo adecuado.

Como se sabe, el cuidado paliativo implica el cuidado físico y emocional de una persona moribunda o víctima de graves sufrimientos tanto por el personal médico como de familiares y amigos. Las claves del éxito de los cuidados paliativos exigen tratar al paciente como una persona, no como un conjunto de síntomas o problemas médicos. La Organización Mundial de la Salud declara que "los cuidados paliativos afirman la vida y consideran que la muerte es un proceso normal; no acelera ni pospone la muerte; proporciona alivio del dolor y el sufrimiento; integra los aspectos psicológicos y espirituales del paciente"18. Su objetivo es mejorar la calidad de vida de la familia y del paciente y brindarles la oportunidad de pasar tiempo de calidad juntos, manejar un cierre adecuado de los asuntos pendientes en sus vidas y para despedirse. Los cuidados paliativos deben tener como objetivo hacer que sea más fácil y más atractivo para la familia y los amigos visitar a la persona moribunda ${ }^{19}$.

En resumen, tanto para el liberalismo igualitario como para la Economía de la Salud la justicia de la despenalización de la eutanasia (sea que se la sustente en el principio de necesidad o de eficiencia) requiere que el Estado asegure condiciones previas de igualdad que permitan responder a quienes temen que la despenalización afecte negativamente a los peor situados.

18 WORLD HEALTH ORGANIZATION: Cancer pain relief and palliative care report. Geneva: WHO; 1990.

19 BUISÁN, R. y DELGADO, J. C.: "El cuidado del paciente terminal”. En: Anales del Sistema Sanitario de Navarra, 30 (Supl. 3), 2007. pp. 103-112. 


\section{Conclusiones}

Los argumentos de justicia distributiva apelan a la idea de que la distribución justa, equitativa y adecuada de todos los derechos, responsabilidades y bienes en la sociedad debe tomar en consideración los problemas de racionamiento sanitario. En un momento en que los sistemas sanitarios de todo el mundo han debido restringir y disminuir progresivamente los gastos siempre crecientes gastos médicos, la pregunta que debemos responder es si ¿es ético financiar un tratamiento costoso e ineficiente para personas con enfermedades terminales con una baja calidad de vida que no quieren vivir?

Analizar la eutanasia y el auxilio médico al suicidio desde la perspectiva de la justicia distributiva nos obliga a considerar si es ético gastar dinero escaso en aquellos que desean morir cuando hay tantos otros que quieren vivir, pero que no tienen los recursos para ello. Y la respuesta, desde el liberalismo igualitario y el utilitarismo es no. No es ni justo ni eficiente, por lo que no está moralmente justificada una legislación como la chilena, que mantiene ambas instituciones al margen de la ley.

\section{BIBLIOGRAFÍA.}

ANDERSON, Elizabeth S.: "What Is the Point of Equality?". En: Ethics 109, 1999.

BEAUCHAMP, WALTERS, KHAN y MASTROIANNI: Contemporary Issues in Bioethics (7th ed.). Thomas Wadsworth, New York, 2008.

BEEAUCHAMP TL y CHILDRESS J.: Principles of Biomedical Ethics (6th ed.). Oxford University Press, New York, 2009.

BENTHAM, Jeremy: An Introduction to the Principles of Moral and Legislation. Eds. Burns y Hart, Methuen, London. 1982.

BUISÁN, R. y DELGADO, J. C.: “El cuidado del paciente terminal”. En: Anales del Sistema Sanitario de Navarra, 30 (Supl. 3), 2007. pp. 103-112.

DANIELS, Norman: Just Health Care. Cambridge University Press, New York.1985.

DWORKIN, Ronald: Sovereign Virtue. The Theory and Practice of Equality. Harvard University Press, London. 2000. 
FRIED, Charles: "Rights and Health Care. Beyond Equity and Efficiency". En: New England Journal of Medicine, Vol. 293, № 5, 1975.

GALVEZ GONZALEZ, Ana María: "Guía metodológica para la evaluación económica en salud: Cuba 2003”. En: Revista Cubana de Salud Pública. 2004. Vol. 30, $\mathrm{N}^{\mathrm{o}} 1$.

KEOWN J.: Euthanasia Examined (1st ed.). Cambridge University Press, Cambridge, 2004.

KYMLICKA, Will. Contemporary Political Philosophy, an Introduction. Second Edition, Oxford University Press, 2002.

WILLIAMS, Bernard. "The Idea of Equality". En: Problems of the Self. Cambridge University Press. 1973.

WORLD HEALTH ORGANIZATION: Cancer pain relief and palliative care report. Geneva: WHO; 1990.

WORLD MEDICAL ASSOCIATION: Medical Ethics Manual [homepage on the internet], 2009. Disponible en: http://www.wma.net/ en/30publications/30ethicsmanual/pdf/ethics_manu al_en.pdf.

ZÚÑIGA FAJURI, A.: "Derechos del paciente y eutanasia en Chile". En: Revista de derecho, UACH, Vol. XXI N², 2008. pp. 111-130.

ZÚÑIGA FAJURI, A.: "La justicia frente a las decisiones médicas". En: Revista de Ciencias Sociales, N 55, 2009. pp. 203-232. 
